\title{
Detection of driver mutations in BRAF can aid in diagnosis and early treatment of dedifferentiated metastatic melanoma
}

\author{
Nasr Alrabadi $\mathbb{1}^{1} \cdot$ Natasha Gibson ${ }^{2} \cdot$ Kendra Curless $^{2} \cdot$ Liang Cheng $^{2} \cdot$ Matthew Kuhar $^{2,3} \cdot$ Shaoxiong Chen $^{2} \cdot$ \\ Simon J. P. Warren ${ }^{2,3} \cdot$ Ahmed K. Alomari $\mathbb{1}^{2,3}$
}

Received: 5 July 2018 / Revised: 18 September 2018 / Accepted: 19 September 2018 / Published online: 12 October 2018

(c) United States \& Canadian Academy of Pathology 2018

\begin{abstract}
Dedifferentiated metastatic melanoma can pose a significant diagnostic challenge, especially if the history of primary melanoma is not known or is remote. $B R A F$ and $N R A S$ mutations are common melanoma driver mutations that are usually sequenced to evaluate for treatment targets. We evaluated whether BRAF and NRAS mutational testing could contribute to the diagnosis of dedifferentiated metastatic melanoma when immunostains are negative. Seven patients with melanoma who had an additional diagnosis of poorly differentiated sarcoma with negative melanocytic immunostains were tested for $B R A F$ and NRAS mutations. Three patients showed identical BRAF mutations in the melanoma and the poorly differentiated sarcoma and hence were re-classified as metastatic dedifferentiated melanoma. In these three patients, there was an average delay of 7 months before appropriate testing, workup and treatment for metastatic melanoma was initiated. Two of these patients currently have stable metastatic disease and show sustained therapeutic response to melanoma-specific treatment including BRAF inhibitors. BRAF mutational analysis should therefore be considered in cases of poorly differentiated sarcoma, especially if there is a known history of melanoma or with unusual localization of disease. The administration of melanoma-specific treatments in such dedifferentiated cases can show therapeutic response, highlighting the importance of rendering accurate diagnoses on such cases.
\end{abstract}

\section{Introduction}

The diagnosis of primary melanoma is usually straightforward except in a subset of patients where the histologic features overlap with various classes of nevi [1]. However, the melanocytic derivation of such lesions is usually apparent, except in some cases with desmoplastic and/or spindle cell morphology [2]. Similarly, establishing a diagnosis of metastatic melanoma, particularly in the setting

Nasr Alrabadi

nnalrabadi@just.edu.jo

$\triangle$ Ahmed K. Alomari

akalomar@iu.edu

1 Department of Pharmacology, Faculty of Medicine, Jordan University of Science and Technology, Irbid, Jordan

2 Department of Pathology and laboratory Medicine, School of Medicine, Indiana University, Indianapolis, USA

3 Department of Dermatology, School of Medicine, Indiana University, Indianapolis, USA of a known history of melanoma is usually not challenging. Occasionally though, melanomas can lose their antigenicity to one or more melanocytic markers, especially in the metastatic setting. This phenomenon is well-documented in the literature and some melanomas can even show aberrant expression of non-melanocytic markers [3]. The diagnostic uncertainty as a result of this phenomenon can potentially lead to significant therapeutic implications. This is particularly true if a metastatic melanoma loses its immunoreactivity to all six of the clinically used melanocytic markers, namely S100, SOX10, Melan-A (MART-1), HMB45, MiTF, and Tyrosinase [4, 5].

$B R A F$ and NRAS mutations are common mutually exclusive driver mutations detected in $\sim 70 \%$ of melanomas $[6,7]$. This underlies the clinical utility of $B R A F$ mutational testing in predicting therapeutic response to $B R A F$ inhibitors in patients with advanced melanoma [8]. However, the use of BRAF and NRAS mutational status for diagnostic purposes has not gained wide popularity [9].

In this report, we aim to highlight the potential impact that mutational testing for common driver mutations such as $B R A F$ and NRAS could have on the diagnosis and early 


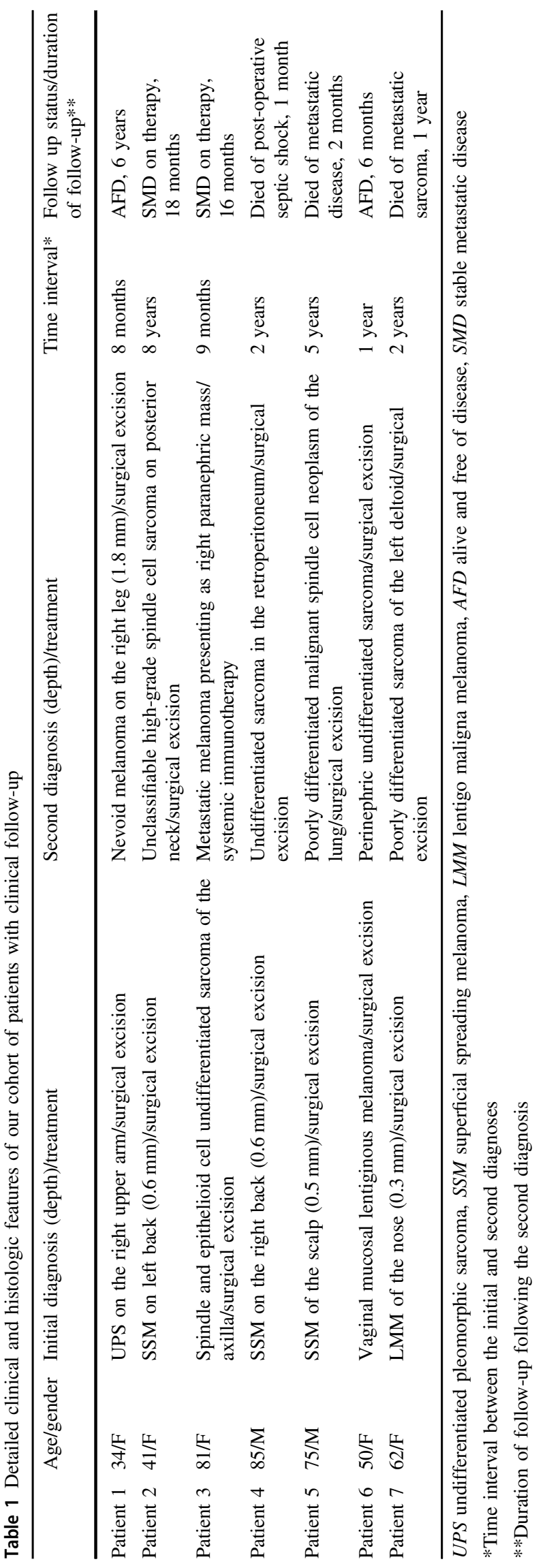

treatment of patients with metastatic melanoma who have been misdiagnosed as poorly differentiated sarcoma due to lack of immunoreactivity to melanocytic markers.

\section{Materials and methods}

\section{Case selection}

The electronic database of the Department of Pathology at Indiana University was searched for patients diagnosed with both melanoma and sarcoma between 1983 and 2018. The pathology reports and slides for sarcoma cases were reviewed and cases with a specific lineage of differentiation or with a specific type of sarcoma were excluded. Patients were included in this study only if they had a diagnosis of melanoma plus a poorly differentiated sarcoma with at least three negative melanocytic markers, including either S100 or SOX10. Additionally, only patients with adequate tissue for further analysis of both the melanoma and sarcoma specimens were included. Institutional review board approval was obtained to conduct this study.

\section{Immunohistochemistry}

Immunohistochemical studies were performed using specific monoclonal antibodies against S100, Melan-A, HMB45, Tyrosinase (DAKO, Denmark), SOX10, and MiTF (CellMarque, USA) on a DAKO Omnis stainer. Formalin-fixed paraffin-embedded tissue sections were used and cut at $4 \mu \mathrm{m}$ thickness. The standard immunoperoxidase technique was followed. The staining was called positive, for any of these markers, if more than $1 \%$ of the cells showed at least moderate intensity staining or if more than $5 \%$ of the cells showed at least weak intensity staining.

\section{BRAF and NRAS mutational testing}

Following light microscopic evaluation and microdissection to increase tumor density, genomic DNA was extracted from six sections of formalin-fixed paraffin embedded material ( $5 \mu \mathrm{m}$ thickness each) using a Qiagen formalin-fixed paraffin embedded DNA extraction kit. The limit of detection was $2 \%$ for the assays. The DNA concentration was determined using the NanoDrop Spectrophotometer prior to testing.

This $B R A F$ assay detects five somatic mutations in the $B R A F$ gene using real-time PCR on the Rotor-Gene Q 5plex HRM instrument. The assay uses amplification refractory mutation system (ARMS) and Scorpions technologies; enabling detection of the following mutations at 
codon 600 of the $B R A F$ oncogene against a background of wild-type genomic DNA: V600E/V600E complex (V600Ec), V600D, V600K, and V600R. The control assay, labeled with FAM, is used to assess the total amplifiable $B R A F$ DNA in a sample. The control assay amplifies a region of exon 3 of the BRAF gene. The primers and Scorpion probe have been designed to amplify independently of any known $B R A F$ polymorphisms. All procedures were performed according to the manufacturer's recommendations (Qiagen Manchester, Manchester, UK).

The therascreen NRAS Pyro Kit was used on the PyroMark Q24 system to detect mutations in codons 12, 13, and 61 of the NRAS gene. The kit consists of two assays: one for detecting mutations in codons 12 and 13 and the other one for detecting mutations in codon 61 . The two regions are amplified separately by PCR followed by pyrosequencing. Sequences surrounding the defined positions serve as normalization and reference peaks for quantification and quality assessment.

\section{Review of literature}

A search for cases of metastatic dedifferentiated melanoma was conducted from Medline-indexed journals in English. Only cases with mutational analysis for both the primary and the secondary/metastatic tumor were included.

\section{Results}

The initial search yielded 55 patients with a diagnosis of both melanoma and sarcoma. Upon review of the pathology slides and reports, 7 patients were identified, where the sarcoma was poorly differentiated, undifferentiated, or unclassifiable. These 7 patients were considered as possible cases where the sarcoma was in reality a dedifferentiated melanoma in which melanoma-specific immunohistochemical markers had been lost. Table 1 summarizes the clinical characteristics of these 7 patients, Table 2 summarizes the results of immunohistochemical and molecular testing, and Figs. 1 and 2 depict representative microscopic images.

The melanomas from these seven patients underwent molecular testing; 5 had BRAF V600E mutation, one had NRAS Q61L mutation and one was wild type for both $B R A F$ and NRAS. Of the five patients with $B R A F \mathrm{~V} 600 \mathrm{E}$ positive melanoma, 3 had an identical $B R A F$ mutation in their poorly differentiated sarcoma. In these three patients, a diagnosis of metastatic melanoma with loss-of-melanoma-specific immunoreactivity was established based on the presence of an identical BRAF mutation in the melanoma and what had previously been diagnosed as poorly differentiated sarcoma.

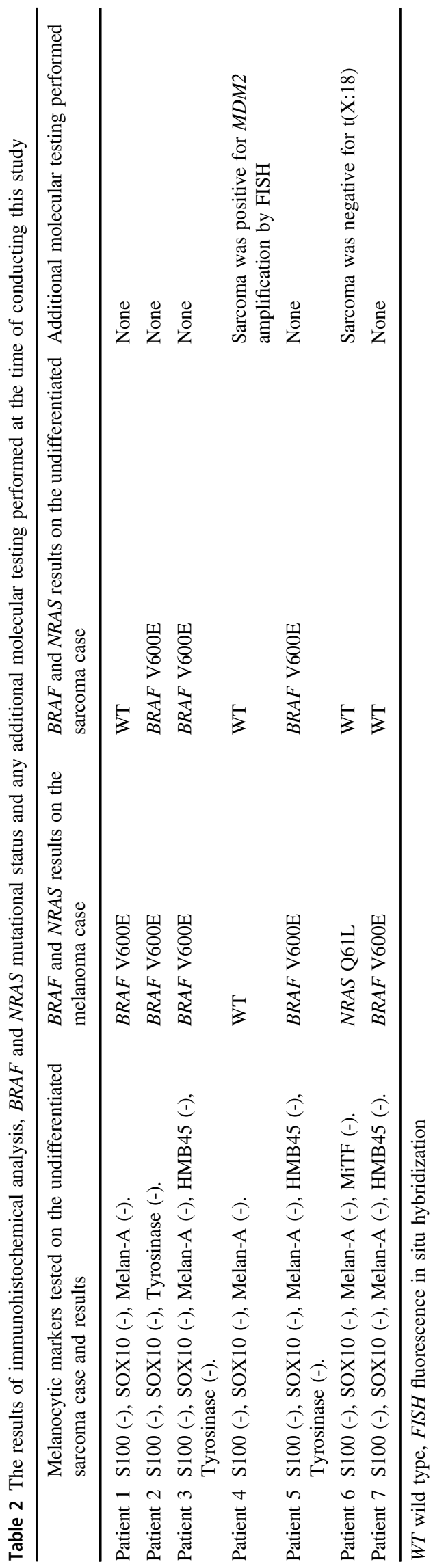


Fig. 1 a $(\times 400, H \& E)$ :

Representative microscopic image of the scalp lesion of patient 5 showing atypical melanocytes arranged in nests and single pagetoid cells consistent with a diagnosis of melanoma. b $(\times 400, H \& E)$ : Representative microscopic image of the lung mass identified 5 years later showing a poorly differentiated spindle cell neoplasm. c $(\times 400, \mathrm{~S} 100)$ and $\mathbf{d}$ $(\times 400$, Melan-A): Both stains are negative in tumor cells from the lung mass. Mutational testing for $B R A F$ identified the V600E mutation in both specimens

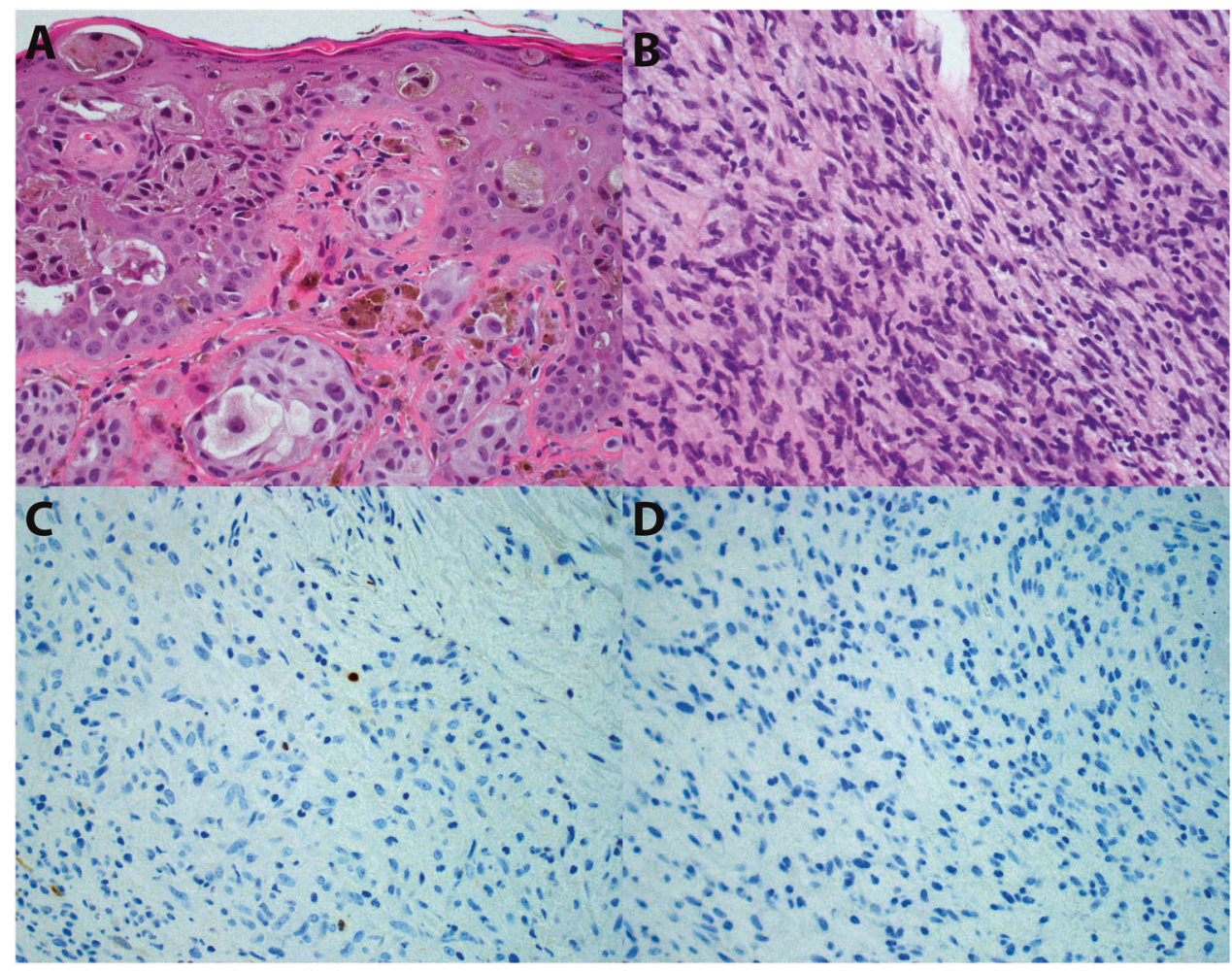

In these three patients, the time interval between the initial diagnosis of "sarcoma" and the performance of metastatic melanoma-appropriate workup including $B R A F$ testing for therapeutic purposes was 6 months (patient 2), 10 months (patient 3), and 5 months (patient 5), leading to a potential average delay in the administration of melanoma appropriate therapeutic interventions of $\sim 7$ months. This interval was longest in the patient with no known history of primary melanoma (patient 3). In follow-up, one of these patients succumbed to widespread metastatic disease while the other two have stable metastatic disease on systemic therapy including $B R A F$ inhibitors after an average follow-up time of 17 months.

The two other patients with BRAF V600E positive melanoma (patients 1 and 7) and the patient with NRAS Q61L mutation in her melanoma (patient 6) had wild type results for BRAF and NRAS in their sarcomas. These three patients had appropriate surgical resection with or without sentinel lymph node biopsy for their melanomas and wide local excision for their sarcoma. Of these patients, one (patient 7) succumbed to widespread metastatic sarcoma while the other two are alive and free of disease. Based on the morphologic appearance and the anatomic location of the sarcoma in patient 6 at the time of conducting this study, additional testing for translocation (X: 18) to rule out the possibility of synovial sarcoma was conducted and was negative.
Finally, patient 4 had wild-type $B R A F$ and NRAS in both his melanoma and sarcoma, limiting our ability to confirm or refute their relationship. However, and based on the location of his sarcoma, it was sent out to a reference lab for fluorescence in situ hybridization testing for $M D M 2$ amplification which was positive, supporting an interpretation of dedifferentiated liposarcoma.

We also reviewed the literature for all cases with an identical mutational profile in the primary and metastatic melanomas where the metastatic tumor was entirely dedifferentiated. To the best of our knowledge, only 7 such cases have been previously reported (Table 3) $[4,5,10]$. None of these reports discussed immediate therapeutic implications or clinical outcomes of such a discovery.

\section{Discussion}

As driver mutations, BRAF and NRAS are commonly identified in various types of melanomas [6, 7]. In our study, they were present in 6 out of 7 cases (86\%). On the other hand, and despite several anecdotal reports, systematic reviews have shown that $B R A F$ or NRAS mutations are exceedingly rare in sarcoma and when present, the patients usually had a prior history of melanoma [5, 11-13]. For instance, out of a total of 204 sarcoma cases tested in two separate studies, three had BRAF V600E mutation and one had NRAS Q601K mutation. All four patients had a prior 
Fig. 2 a $(\times 200, H \& E)$

Representative microscopic image of the axillary mass in patient 3 depicting a poorly differentiated spindle cell neoplasm with nuclear pleomorphism. b $(\times 100, \mathrm{H} \& \mathrm{E})$ Remnants of lymph node tissue are identified in the same specimen showing surrounding fibrosis, inflammation and fat necrosis, suggestive of reactive peritumoral changes. c $(\times 400$, S100), d ( $\times 400$, SOX10), e $(\times 400$, Melan-A), and $\mathbf{f}(\times 400$, HMB45): All four stains are negative in the lesional cells. $\mathbf{g}$ ( $\times 800, H \&$ E): Representative image of the cell block of fine needle aspiration biopsy of the paranephric mass identified 9 months later showing a collection of neoplastic cells with high nuclear to cytoplasmic ratio. h $(\times 400$, SOX10): The lesional cells are positive consistent with a diagnosis of melanoma (Melan-A (not shown) was also positive in the lesional cells). Mutational testing for $B R A F$ identified the V600E mutation in both specimens

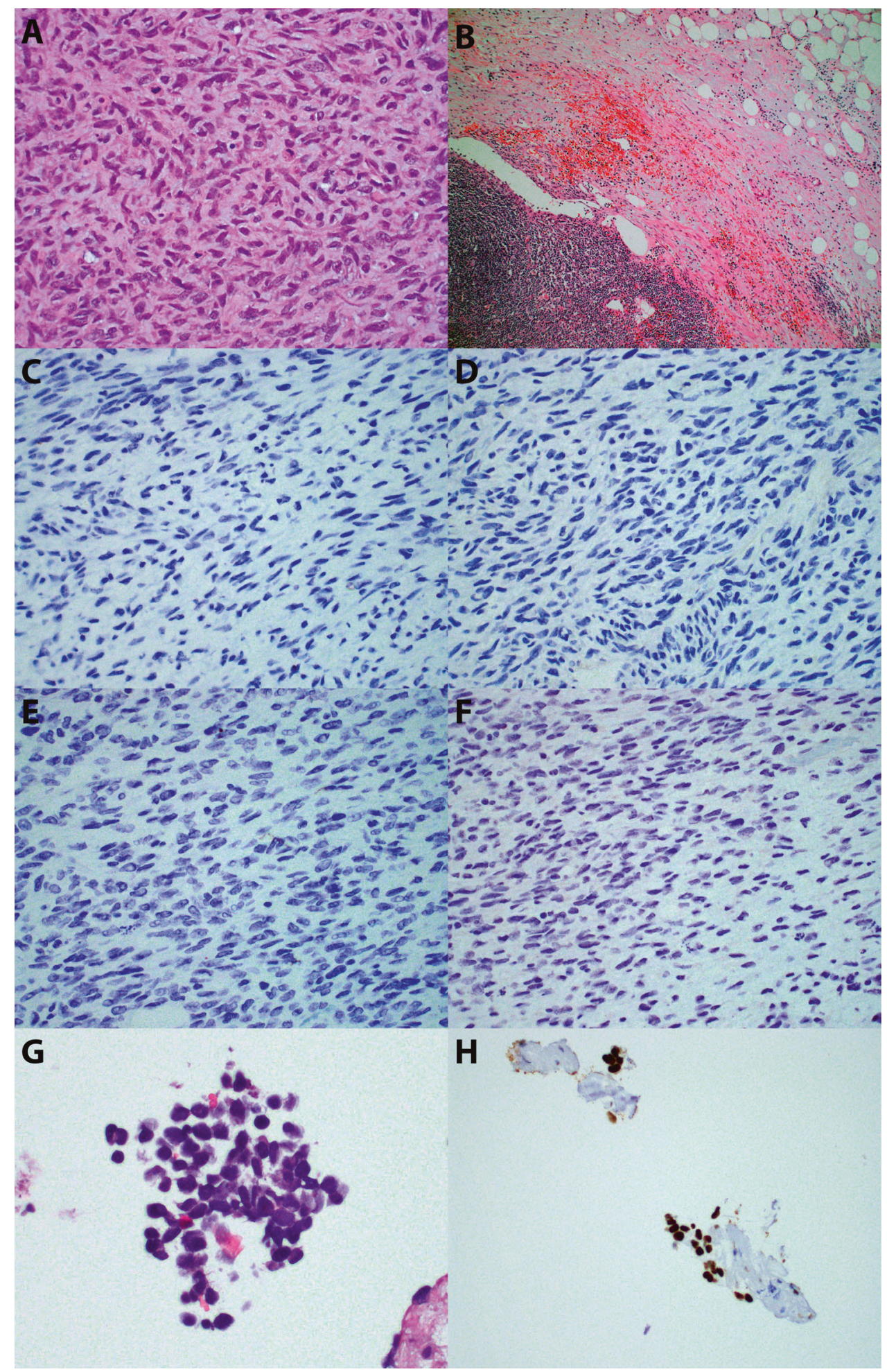

history of melanoma and all were diagnosed histologically as undifferentiated pleomorphic sarcoma [5, 12]. The associated diagnosis of melanoma, the aggressive clinical behavior and the existence of identical $B R A F$ mutations were strongly suggestive of a diagnosis of metastatic melanoma in three of our patients whose lesions had been originally diagnosed as sarcomas. These results emphasize the role of mutational analysis as a diagnostic aid in dedifferentiated metastatic melanoma.

Although several cases of metastatic dedifferentiated melanoma have been previously reported, the diagnosis was usually confirmed by the identification of more 
Table 3 Patients with same mutation in primary melanoma and metastatic dedifferentiated melanoma identified by literature review

\begin{tabular}{|c|c|c|c|c|c|}
\hline Study & \# of patients & Histology of metastasis & $\begin{array}{l}\text { Molecular } \\
\text { findings }\end{array}$ & $\begin{array}{l}\text { Time to metastasis. Range } \\
\text { (years)/average (years) }\end{array}$ & $\begin{array}{l}\text { Follow-up status } \\
\text { (duration) }\end{array}$ \\
\hline $\begin{array}{l}\text { Agaimy et al. } \\
2016\end{array}$ & 4 & $\begin{array}{l}\text { Patient } 1 \text {. UPS with myxoid areas. } \\
\text { Patient 2. RMS \& } \\
\text { teratocarcinosarcoma-like. } \\
\text { Patient 3. SCS } \\
\text { Patient 4. SCS }\end{array}$ & $\begin{array}{l}B R A F \text { V600E } \\
B R A F \text { V600E } \\
N R A S \text { Q61K } \\
N R A S \text { Q61R }\end{array}$ & $0-2 / 1$ & $\begin{array}{l}\text { AWD (N/A) } \\
\text { AWD (N/A) } \\
\text { AWD (N/A) } \\
\text { AWD (N/A) }\end{array}$ \\
\hline $\begin{array}{l}\text { Cipriani et al. } \\
2014\end{array}$ & 1 & UPS & $B R A F$ V600E & $42 *$ & N/A \\
\hline $\begin{array}{l}\text { Bekers et al. } \\
2014\end{array}$ & 2 & $\begin{array}{l}\text { Patient 1. SFT } \\
\text { Patient 2. SFT }\end{array}$ & $\begin{array}{l}\text { NRAS Q61K } \\
B R A F \text { V600E }\end{array}$ & $7-12 / 10$ & N/A \\
\hline
\end{tabular}

UPS undifferentiated pleomorphic sarcoma, RMS Rhabdomyosarcoma, SCS spindle cell sarcoma, SFT solitary fibrous tumor, AWD alive with disease

* The BRAF analysis was not performed on the primary melanoma but rather on a concurrent metastatic lesion with retained expression of melanocytic markers

differentiated foci within the same metastatic lesion with or without additional genetic testing [14]. In contrast, the diagnosis was presumptive in several other reports given the patient's known history of melanoma and/or the identification of oncogenes frequently mutated in melanoma in the metastatic focus alone. However, the added identification of identical mutations in the associated melanoma as shown in this report and a few others (Table 3) provides strong evidence in establishing a diagnosis of dedifferentiated metastatic melanoma. Given the ever-growing availability of $B R A F$ and NRAS testing, pathologists are in a unique position where they can incorporate these molecular markers into their routine practice when faced with undifferentiated neoplasms in a patient with a recent or remote history of melanoma.

In one of our patients, the diagnosis of "sarcoma" preceded the diagnosis of melanoma (patient 3). This was associated with the longest delay in establishing the diagnosis of metastatic melanoma (10 months). It is of note, though, that upon review of the sarcoma-excision specimen, focal areas of lymph node remnants were identified. Since sarcoma metastatic to lymph nodes is highly unusual $[15$, 16], this could provide an additional clue to initiate mutational analysis in the absence of a known history of melanoma. Moreover, in these instances of an undifferentiated malignant neoplasm in a lymph node basin, it is important to consider and perform appropriate workup to investigate the possibility of other metastatic tumors with a $B R A F$ mutation, such as anaplastic thyroid carcinoma, non-small cell lung carcinoma and poorly differentiated colonic adenocarcinoma among others. Nonetheless, detection of $B R A F$ mutation may still be beneficial in guiding $B R A F$-inhibitor therapy in the absence of a definitive diagnosis [17].

There are several recent additions to the armamentarium of agents with survival benefit in the treatment of metastatic melanoma, such as immune checkpoint inhibitors and oncogene-targeted inhibitors $[18,19]$. However, it is increasingly recognized that lower metastatic tumor burden at baseline and hence early diagnosis is an important factor in predicting better response to these agents [20, 21]. Despite rapid deterioration in one patient and an average delay of 7 months, the other two patients are showing continued response and overall stable metastatic disease on systemic therapy including $B R A F$ inhibitors. In contrast, there are very limited effective therapies for advanced stage sarcomas [22, 23].

In addition to $B R A F$ and NRAS mutations, one can project that testing for other less frequent driver mutations in melanomas, such as Kit, NF1, GNAQ, and GNA11 could also be beneficial in this setting, either as part of an expanded panel or in a more stepwise approach following inconclusive results with $B R A F$ and NRAS. Moreover, in two $B R A F$ mutational analysis studies of primary and metastatic melanomas [24, 25], the authors reported a discordant mutational result between different metastatic foci in the same patient in 18.9 and $26 \%$ of cases, respectively. Additionally, 2 out of 25 patients $(8 \%)$ in another study showed $B R A F$ wild type in the metastatic melanoma despite having $B R A F$ mutant primary melanomas [26]. Although detailed review of the literature showed no definitive reported cases of metastatic dedifferentiated melanoma with loss of both melanocytic immunohistochemical reactivity and mutational status, and despite the alternative possibilities of metastasis from a separate primary or false negative mutational testing [26], one can argue that testing of multiple metastatic foci and a more detailed, potentially genome wide, mutational analysis may be necessary in such instances to render a definitive diagnosis and allow patients to benefit from the broader therapeutic repertoire available for metastatic melanoma. Patient 7 in our cohort had a history of $B R A F$ mutated primary melanoma and succumbed to widespread metastatic sarcoma that was $B R A F$ 
wild type. However, no additional tissue was available for analysis to test the hypothesis of potential discordant mutational status between different metastatic foci.

In this study, cases with a specific lineage of differentiation in the sarcoma specimen were excluded from further analysis despite the well-reported phenomenon of heterologous differentiation in melanoma [27]. Nevertheless, this phenomenon appears much more common in the primary rather than the metastatic setting [28]. Additionally, in almost all previously reported cases of heterologous or divergent differentiation in metastatic melanoma, areas of more differentiated melanoma were focally identified $[14,28]$. Still, as we progress into the era of individualized medicine and more detailed molecular characterization of tumors including sarcomas, it is imperative to include driver mutations frequently mutated in melanoma in the evaluation of undifferentiated tumors and potentially also differentiated tumors so as to allow early and accurate identification of dedifferentiated and/or divergent melanomas.

One limitation of this study is the sample size. This is a result of the study design which is restricted to patients with both a melanoma and presumed undifferentiated sarcoma for the purposes of proving the principle. However, we anticipate the findings having diagnostic utility in a wider group of patients such as patients with metastatic melanoma with unknown primary, and potentially in the workup of presumed primary undifferentiated sarcoma where a spindle cell melanoma is a possibility. In addition, although the category of patients that may be assisted by this finding is small, the finding is significant because it also implies the possibility of therapy with $B R A F$ inhibitors.

In summary, the diagnostic dilemma that dedifferentiated metastatic melanoma can create is well-reported in the literature. Using a cohort of patients with both melanoma and a poorly differentiated sarcoma, we provide further evidence to support the role of mutational testing as an aid in diagnosing metastatic melanoma when immunostains are negative. Uniquely, we also reviewed the clinical outcomes of such patients and highlighted the potential impact pathologists can have on timely treatment decisions in such patients.

\section{Compliance with ethical standards}

Conflict of interest The authors declare that they have no conflict of interest.

\section{References}

1. Farmer ER, Gonin R, Hanna MP. Discordance in the histopathologic diagnosis of melanoma and melanocytic nevi between expert pathologists. Hum Pathol. 1996;27:528-31.
2. Andreevscaia O, Theate I, Goossens C, et al. Diagnostic challenge of desmoplastic melanoma. Rare Tumors. 2016;8:5713.

3. Plaza JA, Suster D, Perez-Montiel D. Expression of immunohistochemical markers in primary and metastatic malignant melanoma: a comparative study in 70 patients using a tissue microarray technique. Appl Immunohistochem Mol Morphol. 2007;15: 421-5.

4. Agaimy A, Specht K, Stoehr R, et al. Metastatic malignant melanoma with complete loss of differentiation markers (undifferentiated/dedifferentiated melanoma): analysis of 14 patients emphasizing phenotypic plasticity and the value of molecular testing as surrogate diagnostic marker. Am J Surg Pathol. 2016;40:181-91.

5. Cipriani NA, Letovanec I, Hornicek FJ, et al. BRAF mutation in 'sarcomas': a possible method to detect de-differentiated melanomas. Histopathology. 2014;64:639-46.

6. Hayward NK, Wilmott JS, Waddell N, et al. Whole-genome landscapes of major melanoma subtypes. Nature. 2017;545: 175-80.

7. Ellerhorst JA, Greene VR, Ekmekcioglu S, et al. Clinical correlates of NRAS and BRAF mutations in primary human melanoma. Clin Cancer Res. 2011;17:229-35.

8. Cheng L, Lopez-Beltran A, Massari F, et al. Molecular testing for $B R A F$ mutations to inform melanoma treatment decisions: a move toward precision medicine. Mod Pathol. 2018;31:24-38.

9. Greco FA, Lennington WJ, Spigel DR, et al. Poorly differentiated neoplasms of unknown primary site: diagnostic usefulness of a molecular cancer classifier assay. Mol Diagn Ther. 2015;19:91-7.

10. Bekers EM, van Engen-van Grunsven AC, Groenen PJ, et al. Metastatic melanoma mimicking solitary fibrous tumor: report of two cases. Virchows Arch. 2014;464:247-51.

11. Hyman DM, Puzanov I, Subbiah V, et al. Vemurafenib in multiple nonmelanoma cancers with BRAF V600 mutations. N Engl J Med. 2015;373:726-36.

12. Jin Y, Shima Y, Furu M, et al. Absence of oncogenic mutations of $R A S$ family genes in soft tissue sarcomas of 100 Japanese patients. Anticancer Res. 2010;30:245-51.

13. Je EM, An CH, Yoo NJ, et al. Mutational analysis of PIK3CA, $J A K 2, B R A F, F O X L 2, I D H 1, A K T 1$ and EZH2 oncogenes in sarcomas. APMIS. 2012;120:635-9.

14. Jalas JR, Vemula S, Bezrookove V, et al. Metastatic melanoma with striking adenocarcinomatous differentiation illustrating phenotypic plasticity in melanoma. Am J Surg Pathol. 2011;35: 1413-8.

15. Johannesmeyer D, Smith V, Cole DJ, et al. The impact of lymph node disease in extremity soft-tissue sarcomas: a population-based analysis. Am J Surg. 2013;206:289-95.

16. Stelzner F, Steinau HU, Friedrichs N, et al. Pathways of regional lymph node metastases originating from soft tissue sarcomas. Chirurg. 2013;84:502-10.

17. Mitsis D, Opyrchal M, Zhao Y, et al. Exceptional clinical response to $B R A F$-targeted therapy in a patient with metastatic sarcoma. Cureus. 2015;7:e439.

18. Atkinson V. Recent advances in malignant melanoma. Intern Med J. 2017;47:1114-21.

19. Azijli K, Stelloo E, Peters GJ, et al. New developments in the treatment of metastatic melanoma: immune checkpoint inhibitors and targeted therapies. Anticancer Res. 2014;34:1493-505.

20. Menzies AM, Long GV. Dabrafenib and trametinib, alone and in combination for $B R A F$-mutant metastatic melanoma. Clin Cancer Res. 2014;20:2035-43.

21. Espinosa E, Marquez-Rodas I, Soria A, et al. Predictive factors of response to immunotherapy-a review from the Spanish Melanoma Group (GEM). Ann Transl Med. 2017;5:389.

22. Wagner MJ, Amodu LI, Duh MS, et al. A retrospective chart review of drug treatment patterns and clinical outcomes among patients 
with metastatic or recurrent soft tissue sarcoma refractory to one or more prior chemotherapy treatments. BMC Cancer. 2015;15:175.

23. Italiano A, Mathoulin-Pelissier S, Cesne AL, et al. Trends in survival for patients with metastatic soft-tissue sarcoma. Cancer. 2011;117:1049-54.

24. Heinzerling L, Baiter M, Kuhnapfel S, et al. Mutation landscape in melanoma patients clinical implications of heterogeneity of BRAF mutations. Br J Cancer. 2013;109:2833-41.

25. Yancovitz M, Litterman A, Yoon J, et al. Intra- and inter-tumor heterogeneity of $B R A F(\mathrm{~V} 600 \mathrm{E}))$ mutations in primary and metastatic melanoma. PLoS ONE. 2012;7:e29336.
26. Bradish JR, Richey JD, Post KM, et al. Discordancy in BRAF mutations among primary and metastatic melanoma lesions: clinical implications for targeted therapy. Mod Pathol. 2015; 28:480-6.

27. Banerjee SS, Eyden B. Divergent differentiation in malignant melanomas: a review. Histopathology. 2008;52:119-29.

28. Saleh J, Wang ML, Harms PW, et al. Malignant melanoma with osteosarcomatous differentiation in a lymph node metastasis. J Cutan Pathol. 2018. https://doi.org/10.1111/cup.13283. [Epub ahead of print]. 